\title{
Game Theoretic Approach to Spectrum Resource Sharing and Pricing in Cooperative Cognitive Radio Networks
}

\author{
Guopeng Zhang ${ }^{1}$, Jie $\mathrm{Gu}^{2}$, Shuo Xiao ${ }^{1+}$, Jun Deng ${ }^{1}$, and Jinbai Zhao ${ }^{1}$ \\ ${ }^{1}$ School of Computer Science \& Technology, China Univ. of Mining \& Tech., Xuzhou, China, 221116 \\ ${ }^{2}$ Department of Fundamental Courses, Xuzhou Air Force College, Xuzhou 221001, China
}

\begin{abstract}
The current game models for Cognitive Radio (CR) networks, such as auction mechanisms and noncooperative games are always used separately and independently, which complies with the simple onelayer dynamic spectrum access (DSA) scenario well. However, integrated game models which jointly consider the negotiation and cooperation between the primary users (Pus) and second users (SUs), and the benefits of individual PUs and SUs in the newly emerged relaying based CR networks has been very rare. The design of the integrated game model has to consider the specific features of the intra-layer competition and the cross-layer negotiation in the new CR scenarios, simultaneously. The layered and cross-layered DSA schemes and related scheduling algorithms research will provide the achievement and outcomes for cooperative relaying and overlay based CR networks. The advantage of the new merging technology which combines cooperative relaying and CR is superior to the pure spectrum-transfer based CR technology nowadays in terms of QoS support and high spectrum and energy efficiency.
\end{abstract}

Keywords: cognitive radio networks, dynamic spectrum access, game theory, cross layer design, convex optimization.

\section{Introduction}

Wireless communication is undoubtedly one of the most important technologies in our modern life. Most wireless communication systems require a frequency band in the radio spectrum for their operation. However, the radio spectrum is a scarce resource. Under the conventional spectrum management policy, each wireless system is assigned a fixed license for exclusive use of certain frequency band. With the deployment of more wireless systems, more licenses or exclusive frequency bands have been allocated. Since the radio spectrum is limited, there arises the so-called "spectrum scarcity" problem, which significantly holds back the further development of wireless communications.

On the other hand, study has shown that the usage efficiency of the radio spectrum is typically low. In a study conducted by QinetiQ for Ofcom [1], it was reported that only 15\%-80\% of the spectrum are being used. There are many parts of the radio spectrum called "spectrum holes" where the frequency bands are not being occupied at specific times in specific locations. To tackle this problem, new changes in the spectrum policy have been introduced by regulators around the world. For example, in the US, FCC has opened the licensed TV spectrum to unlicensed users [2]. In the UK, Ofcom proposes to increase the percentage of the market-based bands from $0 \%$ to $71.5 \%$ [1]. Spectrum license holders are allowed to rent their licensed bands out when they are not using them to increase their revenues. All these policy changes aim to maximize the social benefits of the radio spectrum. Cognitive radio (CR) [3] is emerging as a key technological enabler of these changes to solve the problem of "spectrum scarcity" as well as to increase the usage efficiency of the radio spectrum.

\footnotetext{
+ Corresponding author. Tel.: + 8618705216712 .

E-mail address: sxiao@cumt.edu.cn
} 
With the development of CR technologies, dynamic spectrum access (DSA) [4] becomes a promising approach to increase the efficiency of spectrum usage, which allows unlicensed secondary users (SUs) to dynamically access the licensed bands of legacy spectrum holders, i.e., primary users (PUs) on a negotiated or an opportunistic basis. In overlay-based CR networks [5-6], PUs are assumed to be aware of the existence of SUs which are using their licensed frequency bands. However, PUs have higher priority on accessing the channel and have the right to improve their own revenue by charging SUs for using their licensed frequency bands.

In CR-based DSA environments, there exists multiple licensed PUs (operate on different frequency bands) and multiple unlicensed SUs. Each PU and each SU serves multiple user connections. A PU is willing to sell some portion of the available idled spectrum resource to multiple SUs at a certain price. And, a PU also prefers to achieve the highest profit by setting the optimal values of the prices. Thus, the PUs and the SUs just constitute respectively the first-layer, i.e., the seller-layer, and the second-layer, i.e., the buyer-layer of the DSA system. In an infrastructure based CR network, the base station (BS) or the access point (AP) in each PU and each SU governs the radio transmission on the occupied spectrum.

Due to channel fading, direct transmission from the primary transmitter (PT) to the primary receiver (PR) of a PU is sometimes severely damaged and thus suffers bad performance in terms of data rate or outage probability. In such a situation, if PUs have heavy traffic loads, they will have no idled frequency band to lease to the SUs. Motivated by the recently emerged physical layer technology, referred to as cooperative relaying, if some of the SUs which have better channel conditions are leveraged as the cooperative relays for the primary transmission, the transmission rate of the PU can be dramatically increased by exploiting cooperative diversity. As the PU's rate is increased to serve certain amount of primary traffic, spectrum resource occupied by the transmission of the PU is decreased, and the SUs could gain more opportunity to access the wireless channel of the PU to transmit more data. As a result, by exploiting the cooperation, both the PUs and SUs can increase their own interest and a win-win situation is achieved. This kind of cooperative relaying based CR systems is called cooperative cognitive radio networks (CCRNs) in the advocated work [7].

Traditional DSAs for CR networks do not consider the cooperation between PUs and SUs. The competition among the PUs/sellers to attract the SUs/buyers is always modeled as a simple pricing-based noncooperative game. And, the gaming is expected to reach a stability point (called the Nash equilibrium (NE)). At the NE, no PU can increase his/her profit by choosing a different pricing strategy, given other PUs' pricing strategies. Hence, in traditional DSA environments, the spectrum demands of SUs only depend on the price charged by the related PUs.

However, this paper considers the cooperative relaying based two-layer multi-PU and multi-SU CCRNs, where the allocated spectrum resource to a SU should be divided into the following two parts: the first part which is used to relay the traffic of the related PUs, and, the second part which can be used by the SU to transmit its own traffic. It also means that part of the data of a PT can be transmitted to its PR in a cooperative manner, while the left part data of the PT can only be transmitted to the PR directly. Hence, DSAs for the considered two-layer cooperative relaying based CCRNs should jointly consider the following multiple aspects: (1) the spectrum prices charged by PUs, (2) relaying channel conditions from SUs to PUs, (3) utility functions for SUs which represent the preference of SUs over some spectrum resources, and (4) quality of service (QoS) demands, e.g., minimum rate requirements of PUs. Moreover, if the QoS demands of SUs are also taken into account (in traditional DSAs, SUs are always treated as best effort (BE) users, and, thus, the QoS demands of SUs are not considered), all those factors together will make the DSAs for the twolayer cooperative relaying based CCRNs become more complicated to deal with.

In this paper, we will investigate and provide an overall solution to tackle the addressed DSA problems for the considered two-layer cooperative relaying based CCRNs. Taking explicitly account of the QoS demands of all the PUs and SUs, the detailed objective of this paper includes (1) an effective pricing mechanism to coordinate the competition among the PUs, (2) a fair and efficient resource allocation scheme for the SUs and (3) an incentive mechanism [8] to guide the SUs to cooperate with the PUs (through cooperative relaying) and to encourage the PUs to release more spectrum resource for the SUs. Through 
further negotiation with PUs, the SUs could obtain more spectrum resources from the PUs to transmit their own traffics. Hence, on condition that the QoS demands of the PUs and SUs are guaranteed simultaneously, the scarcity spectrum resource can be more efficiently utilized. The above research objectives demonstrate that our DSA schemes are energy efficient which meet the green radio requirements of the next generation networks (NGNs). Moreover, distributed implementations with low network overhead and low computational complexities are also essential for the designed DSA schemes.

The main methodology adopted in this paper to develop the DSA schemes for the two-layer cooperative relaying based CCRNs is game theory [9], [10], as game theory is a natural and powerful tool to study the situations of conflict and cooperation. And, to solve the optimization problems derived from the proposed game models, several optimization theories, e.g., convex optimization theory, dual theory, multiobjective optimization theory and particle swarm optimizer (PSO) will also be studied and implemented in this paper. They are used to develop rapid convergence algorithms to reach the game results in a distributed way.

\section{Problem Formulation}

The prime objective of this paper is to investigate incentive mechanisms to stimulate cooperation between multiple PUs and multiple SUs in the new emerged cooperative relaying based CCRN paradigm, and, as a result, to provide related fair and efficient DSA schemes. In particular, the following three innovative technical concepts will be investigated:

- Pricing strategies for the PUs in the seller-layer to coordinate the competition for the buyers/SUs which could bring the maximum revenue for each PU.

- Incentive mechanisms in the buyer-layer competition which could stimulate the SUs to share the allocated spectrum resource fairly and efficiently.

- Cross-layer negotiation mechanism between the seller-layer and the buyer-layer to encourage the PUs to release more spectrum resource for the SUs through cooperative relaying.

To this end, the following measurable technical objectives will be addressed:

- To design data-frame structures for OFDMA, TDMA, of FDMA based two-layer cooperative relaying used CCRNs. The designed frame structures could accommodate both the spectrum resource and the energy resource for a PU (or an SU). This is essential as a rate-related utility function with concrete physical meaning is expected for the game theory based analysis.

- Based on the designed frame structures and utility functions, to design and implement pricing mechanisms to coordinate the competition for the buyers/SUs in the seller-layer of the system.

- To develop distributed price-updating algorithms for the PUs that converges to the optimal equilibrium prices of the seller-layer competition and requires only limited network knowledge.

- To design QoS-aware DSAs for the SUs in the buyer-layer competition. Considering a wireless user is always "selfish" to consume the resource solely to maximize its own benefit/utility. Thus, appropriate fairness criterions, e.g., the max-min rule and the proportional fairness rule should be adopted to guide the SUs to share the allocated spectrum resource fairly and efficiently.

- Jointly considering the benefits of the PUs and SUs, to design and implement a distributed cross-layer resource scheduler to stimulate the SUs to cooperate with the PUs through traffic relaying and to encourage the PUs to release more spectrum resource for the SUs.

- To design and implement other supporting algorithms or techniques that underpin the operation of the above intra-layer and cross-layer algorithms. And, to evaluate the proposed network system and its algorithms using analytical modeling and simulations.

\section{Research Methodology}

In general, the paper will start with a design of overall system architecture. Following on this, first, the pricing strategies and incentive mechanisms will be designed and modelled using multiple particular game models. By adopting different optimization theories, distributed algorithms and protocols with low complexity will be developed to search for the solutions of the games. The overall performance of the new system is to be evaluated theoretically, and, therefore, to find out some theoretical boundaries of some 
important parameters. Thereafter, the designed algorithms/ protocols are to be implemented in the simulator. Based on carefully simulation design, the performance of the system is to be compared against both its theoretical results and other representative benchmarks. More refinements are to be carried out based on the evaluation results. The slightly detailed methodology corresponding to each research objectives are as follows:

- The study of the pricing strategies for PUs. It can be analyzed in the following two separate cases. In the first case, the number of the idled spectrum resource of PUs is assumed to be much larger than that the spectrum demands of SUs. It just corresponds to a buyer's market situation where supply exceeds demand, which allows the buyers/SUs of product/spectrum to have greater control over prices. The situation can be dealt with by using the classical Bertrand game theory. The opposite of a buyer's market is the seller's market, where the spectrum demands of SUs exceed the supplies of PUs. In such situations, PUs have greater control over prices. Hence, we can deal with this situation by using the classical Stackelberg game theory.

- The research of QoS-oriented DSAs for the SUs which share the idled spectrum resource of the same PUs. Especially, the following two basic issues, i.e., (1) how to stimulate the PUs to split extra more spectrums to multiple SUs (through cooperative relaying) while sustain the minimum rates of the PUs and (2) how to allocate the newly obtained spectrum from the PUs efficiently and fairly among multiple competitive SUs should be carefully addressed.

- The research of the fast convergence algorithms to solve the proposed game problems. According to whether the games are distributed games or centralized games, the investigated algorithms fall into the following two categories. The first category orders distributed implementation, e.g., the price-update algorithm for the distributed PUs. By appropriate utility function designing, convex problems can be derived from the game model. Thus, convex optimization theory and dual theory can be applied to this case. The second category of algorithms are unnecessarily distributed, e.g., in a cooperative DSA game for the SUs, a PU can serve as a central gathering point, via which, the distributed SUs can negotiate and cooperate in making the decisions on the spectrum usage. The DSA strategies for the SUs can be computed at the PU and then be conveyed to the SUs via dedicated feedback channels. Thus, the messages needed to be communicated among the SUs to coordinate the DSAs are greatly reduced. Low complexity PSO based algorithms which do not need the convex objective function of the game problems can be used in such cases.

- The research of a QoS-guaranteed adaptive group admission control (AC) and handover schemes for the two-layer CCRNs. Based on the Hidden Markov Model (HMM), the PUs can predict group handover and prepare the necessary resources for the SUs, and then improve the QoS parameters of all the PUs and SUs during handover process. The AC and handover schemes also need the cross-layer resource scheduling mechanism to support. Hence, we will introduce a cross-layer scheduling mechanism and design a sound and effective cross-layer scheduling algorithm, which can enhance the whole system throughput and user fairness, and, at the same time, decrease the outage probabilities of all the PUs and SUs on the condition of users' QoS.

Pricing strategies and DSAs for CR networks have attracted increasing research interest but the work which concentrates on the multi-hop relaying based two-layer CR networks has been rare. In particular, we promote the combining usage of cooperative relaying and other advanced wireless communication technologies, e.g., OFDMA and MIMO technologies that are specific to the green radio (i.e., energy efficient) oriented and QoS-aware DSAs in the considered CR environments.

Layered and cross-layered DSAs are proposed in contrast to traditional one-layer DSAs. The competition/cooperation and negotiation within the seller/PU layer and the buyer/SU layer, and between these two layers provide a natural backing on which to build up the layered competition/cooperation, and the cross-layered negotiation mechanisms. The layered and cross-layered DSAs coordinate with each other to provide more precise and nearer-optimal resource allocation at a cost of a small controllable increased computational complexity and network overhead comparing with the traditional one-layer DSAs.

The current game models for CR networks, such as auction mechanisms and noncooperative games are always used separately and independently, which complies with the simple one-layer DSA scenario well [4- 
5]. However, integrated game models which jointly consider the negotiation and cooperation between the PUs and SUs, and the benefits of individual PUs and SUs in the newly emerged relaying based CR networks has been very rare. The design of the integrated game model has to consider the specific features of the intralayer competition and the cross-layer negotiation in the new CCRN scenarios, simultaneously.

The designed game models contain a number of work packages that aim at the research for distributed strategy selection topics. With the application of various theories from different angles, such as convex optimization theory, heuristic algorithms, transportation, queuing theory, probability theory, and flow control, etc, these topics are to extend the research scope and efficiency, and to make them accessible in the most trusted and natural way.

\section{Conclusions}

CR based DSA is an enabling technique that promises to overcome the problem of spectrum scarcity caused by the current way of fixed spectrum allocation. The advanced wireless communication technologies, such as cooperative relaying, OFDMA, and MIMO will provide potential benefits for the DSA in whether multi-hop ad hoc or centralized infrastructure based CR networks. All of these enable intelligent detection of the spectrum holes that are temporarily unused by licensed PUs and more important, to dynamically utilize those spectrums efficiently. The proposed pricing strategy and DSAs ensure the fairness amongst the traffics of the PUs and SUs. It also provides green radio oriented, i.e., energy efficient applications for the environmental protection purpose. The demonstrative system based on system architecture and open source software are implemented by enabling technologies for CR service creation, allowing rapid service deployment, testing independently of specific execution platforms, and guaranteeing interoperability via the development of a structured logical CCRN platform architecture.

\section{Acknowledgements}

This work was supported in part by the National Nature Science Funds of China under Grant 61471361, and by the Natural Science Foundation of Jiangsu Province of China under Grant BK20150193.

\section{References}

[1] Ofcom, "Spectrum framework review: a consultation on Ofcom's views as to how radio spectrum should be managed," Nov. 2004.

[2] FCC, "Notice of proposed rule making," ET Docket No. 04-113, May 2004.

[3] H. Zhang, C. Jiang, et al, "Resource allocation for cognitive small cell networks: a cooperative bargaining game theoretic approach", IEEE Transactions on Wireless Communications, vol. 14, no. 6, pp. 3481-3493, June 2015.

[4] B. Wang and K. J. R. Liu, "Advances in cognitive radio networks: a survey." IEEE Jounal of Selected Topoics in Signal Procession, vol. 5, no. 1, 2011, pp. 5-23.

[5] D. Niyato and E. Hossain, "Competitive spectrum sharing in cognitive radio networks: a dynamic game approach," IEEE Trans. Wireless Communications, vol. 7, no. 7, 2008, pp. 2651-2660.

[6] D, Niyato, et al, "Competitive pricing for spectrum sharing in cognitive radio networks: dynamic game, inefficiency of Nash equilibrium, and collusion. IEEE J. Select. Areas Commun., vol. 26, no. 1, 2008, pp. 192-202.

[7] H. Zhang, C. Jiang, J. Cheng, and V. C.M. Leung, "Cooperative interference mitigation and handover management for heterogeneous cloud small cell networks ", IEEE Wireless Communications, vol. 22, no. 3, pp. 92-99, June 2015.

[8] L. Duan, L. Gao and J. Huang, "Contract-based cooperative spectrum sharing," in proc. IEEE New Frontiers in Dynamic Spectrum Access Networks (DySPAN), 2011, pp. 399-407.

[9] CG. Y, et al, "Optimal power control for cognitive radio networks under coupled interference constraints: a cooperative game-theoretic perspective. IEEE Trans. Vehicular Technology, 2010, vol. 59, no. 4, pp. 1696-1906.

[10] Q. Ni and C. C. Zarakovitis. "Nash bargaining game theoretic scheduling for joint channel and power allocation in cognitive radio systems", IEEE JSAC, 2012, vol. 30, no. 1, pp. 70-81. 